\title{
Heart failure in real world
}

\author{
Claudio Giumelli, ${ }^{1}$ Azio Reverzani, ${ }^{1}$ Riccardo Volpi, ${ }^{2}$ Giuseppe Chesi ${ }^{1}$ \\ ${ }^{1}$ Internal Medicine, C. Magati Hospital, Scandiano AUSL Reggio Emilia; ${ }^{2}$ Medical Clinic, Univeristy Hospital of Parma, Parma, \\ Italy
}

\begin{abstract}
The heart failure (HF) is one of the greatest problems of public health with increasing epidemiological importance. In the present study we analyzed a population of 299 patients, consecutively admitted to hospital, whose diagnosis of HF was verified retrospectively. In our analysis we considered underlying heart diseases, comorbidities, ejection fraction, presence of atrial fibrillation and pleural effusion, values of NT pro-BNP and causes of destabilization precipitating HF. The mean age of our population was 81 years. Patients with preserved systolic function were 145 (61\% of the total, 59 male and 86 female). 166 patients (69\% of the total) had hypertensive heart disease and 211 had hypertension ( $88 \%$ of the total). Patients with pleural effusion were 108 (46\% of total). In the total population 102 patients (43\%) had from 3 to 5 comorbidities, 169 patients $(71 \%)$ had at least 2 comorbidities and only 4 patients $(1.7 \%)$ had no comorbidities. The collected data highlight the complexity of patients with HF, often due to advanced age and a high number of comorbidities.
\end{abstract}

\section{Introduction}

Heart failure (HF) is one of the most important problems of public health with an increasing epidemiological impact in recent years. HF represents a disease with incidence and prevalence still rising, characterized by high morbidity and mortality and heavy burden in terms of costs for public health system.

$\mathrm{HF}$ can be defined as an alteration of the structure or function of the heart leading to inability to ensure an appropriate tissue perfusion or to permit it only through a filling pressure increase. ${ }^{1}$ Clinically, HF presents as a syndrome with characteristic symptoms

Correspondence: Giuseppe Chesi, Internal Medicine, C. Magati Hospital, Scandiano AUSL Reggio Emilia, via Martiri della Libertà 6, 42019 Reggio nell'Emilia, Italy.

Tel.: +39.0522.850328.

E-mail: chesig@ausl.re.it

Key words: Heart failure; comorbidity; retrospective analysis; hospitalized patients.

Conflict of interest: the authors declare no potential conflict of interest.

Received for publication: 9 October 2014.

Revision received: 7 November 2014.

Accepted for publication: 14 November 2014.

This work is licensed under a Creative Commons Attribution NonCommercial 3.0 License (CC BY-NC 3.0).

(C) Copyright C. Giumelli et al., 2015

Licensee PAGEPress, Italy

Italian Journal of Medicine 2015; 9:356-361

doi:10.4081/itjm.2015.550 (dyspnea and orthopnea in particular, asthenia and fatigue) and signs (peripheral edema, jugular venous distension, rales). However, the diagnosis of HF is not always easy, since many of the symptoms are also common to many other diseases. The demonstration of underlying heart disease is therefore crucial both for diagnostic and for therapeutic approach.

The prevalence of HF in the general adult population is between $2 \%$ and $3 \%$ and increases significantly in patients over 75 years of age (the prevalence of HF in the age group between 70 and 80 years ranges between 10 and $20 \%)^{2}$. The increase in the overall prevalence is justified mainly by the ageing of the population and the prolonged survival of patients with hypertension and ischemic heart disease. HF causes $5 \%$ of total hospitalization, it is found in $10 \%$ of hospitalized patients and is source of $25 \%$ of the cost of the National Health System (a large part of this cost is due to hospitalizations). ${ }^{3}$ Patients with HF have a 5year mortality of about $55 \%$, non-cardiovascular causes of death are rising especially in patients with preserved systolic function. ${ }^{4}$

There are many conditions that can lead to HF and they have a different distribution in different parts of the world. Approximately $50 \%$ of patients with HF have a reduced left ventricular ejection fraction (LVEF). Ischemic heart disease, that is often associated with hypertension and diabetes mellitus, leads to about two-thirds of HF with reduced LVEF. The valvular diseases account for about $10 \%$ of the causes of HF with reduced LVEF with a decreasing incidence in Western countries because of the always easier identification and subsequent surgical treatment. There are also many other conditions leading to HF with reduced LVEF: myocarditis, alcohol abuse, cardio-toxicity chemother- 
apy in addition to idiopathic dilated cardiomiopathy. ${ }^{5}$ The HF with preserved LVEF, also called diastolic HF, presents a different etiology, epidemiology and prognosis. ${ }^{6}$ Patients with diastolic HF are more likely to be older, female, obese and are less likely to have ischemic heart disease but are more often affected by high blood pressure and atrial fibrillation.?

The diagnosis of HF can be difficult, especially in the early stages of disease. In fact many of its symptoms are non-specific. Symptoms that are more specific for HF (orthopnea and paroxysmal nocturnal dyspnea) are often present only in patients with clear clinical pictures. ${ }^{8}$ Many of the signs of HF are correlated to sodium and water retention (especially pitting ankle edema) and are often non-specific or non-detectable if the patient is already receiving diuretics. ${ }^{9}$ In any case, symptoms and signs of HF are often difficult to identify and this is even more difficult in obese patients, patients with chronic lung disease and in elderly. ${ }^{10}$ It is therefore important to use the anamnestic information in order to detect the presence of risk factors or past medical conditions that might have led to an alteration in the structure or function of the heart and their consistency with the patient's symptoms. There are several laboratory and instrumental investigations useful in the diagnosis of HF. Electrocardiogram, echocardiogram and chest $\mathrm{x}$-ray are the most useful tools in patients with suspected HF. In particular echocardiogram allows to obtain useful information regarding volumes, left ventricular systolic and diastolic function, wall thickness and presence of valvular disease. ${ }^{11}$ Among laboratory investigations, measurement of natriuretic peptide (BNP, NT pro-BNP) has acquired a certain value in the differential diagnosis of HF. In the presence of natriuretic peptide's values within the normal limits it is allowed to rule out diagnosis of HF with an accuracy of $95 \% .^{12}$ Several other laboratory tests including blood count and renal function are important to guide therapeutic approach and determinate the severity of the HF.

The prognosis of HF is influenced by several factors among which the most important appear to be age and comorbidity. ${ }^{13}$ The assessment of the prognosis and, in particular the identification of comorbidities, have a primary importance for the classification of patients with $\mathrm{HF}$ and for the management of therapies. ${ }^{14}$ The aim of treatment is to reduce symptoms and signs of HF, improve the quality of life, reduce mortality and prevent hospitalization. ${ }^{15}$ The therapy of HF is based, wherever possible, on removing underlying diseases (myocardial revascularization in ischemic heart disease, corrective valvular surgery, etc.). The therapeutic approach must be based on educational measures (salt and water intake, etc.). Drug therapy includes both symptomatic drug treatment (diuretics) and treatment with drugs that can improve survival [ $\beta$ - blockers, angiotensin converting enzyme-inhibitors (ACE-I) an renin-angiotensin-aldosterone system (RAAS) blockers]. In selected cases minimally invasive treatments (myocardial resynchronization with pacemaker) can be used or, in younger patients with particularly severe heart disease, heart transplantation may be performed.

\section{Aim of the study}

In this study we wanted to analyze a real population of patients consecutively admitted to hospital whose diagnosis of HF was verified retrospectively. The aim was to obtain an image from the real world of a disease that will have an increasing importance in terms of care as well as costs for our National Health System. We wanted to verify in a more homogeneous cohort of patients compared to those of previous multicentric studies, the real characteristics of patients with heart failure hospitalized in a Department of Medicine.

\section{Materials and Methods}

Our work is based on a retrospective survey conducted on a population of patients consecutively admitted to the Department of Internal Medicine of a proximity hospital, with diagnosis of HF in the calendar year 2012. To gather data we analyzed all clinical records as well as discharge letters of every single patient. The diagnosis of HF was verified including in the analysis only patients who had either symptoms and signs characteristic of HF as well as instrumental findings that confirm the presence of underlying structural heart disease. All patients with NT-proBNP values below $500 \mathrm{pg} / \mathrm{mL}$ were excluded.

Regarding underlying heart disease we have included: ischemic heart disease, hypertensive heart disease, valvular heart disease and idiopathic dilated cardiomyopathy.

We considered as ischemic heart disease all patients who had history of previous acute coronary events or episodes of angina pectoris. ${ }^{16}$ As part of hypertensive heart disease we have included patients with history of hypertension and instrumental findings documenting the presence of concentric left ventricular hypertrophy with evidence of diastolic dysfunction. ${ }^{17}$ In the group of idiopathic dilated cardiomyopathy we considered patients with echocardiographic finding of ventricular dilatation associated with impaired left ventricular function and with previous coronary angiography negative for ischemic heart disease. ${ }^{18}$ In the group of valvular heart disease we considered aortic and mitral stenosis and regurgitation. In our analysis we included severe and moderate degree of valvular disease documented by 
echocardiogram performed during hospitalization or within 12 months before admission. For aortic stenosis was considered the trans-valvular gradient equal or greater than $30 \mathrm{mmHg}$. For mitral stenosis was considered mean trans-valvular gradient equal or greater than $5 \mathrm{mmHg}$. For aortic and mitral regurgitation we included almost $++/++++$ color-doppler-degrees.

Among comorbidities we considered: hypertension, diabetes mellitus, chronic obstructive pulmonary disease (COPD), anemia and kidney failure.

For hypertension we considered history of hypertension and/or treatment with antihypertensive drug. For diabetes we considered patients who had previous diagnosis of diabetes made according to the universally accepted criteria and/or antidiabetic treatment or with glycated hemoglobin measurement during the hospitalization greater than $6.5 \%$. For COPD we considered only patients who had a diagnosis established by spirometric tests according to the criteria of the Global Initiative for Chronic Obstructive Lung Disease (GOLD) project. Anemia was defined according to the World Health Organization (WHO) criteria: hemoglobin less than $12.5 \mathrm{~g} / \mathrm{dL}$ in males and less than $11.5 \mathrm{~g} / \mathrm{dL}$ in females. Renal failure was considered with a glomerular filtration rate less than or equal to $45 \mathrm{~mL} / \mathrm{min} / 1.73 \mathrm{~m}^{2}$ corresponding to a stage $3 \mathrm{~B}$ according to National Kidney Foundation produces clinical practice guidelines through the National Kidney Foundation/Disease Outcomes Quality Initiative (NKF/DOQI).

We also evaluated the number of comorbidities present simultaneously in hospitalized patients with HF.

Ejection fraction was calculated by using the biplane Simpson method and in agreement with the literature data we have defined as preserved systolic function LVEF values greater than or equal to $50 \%$.

In the group of Atrial fibrillation we included patients whose AF had not appeared during hospitalization but it was pre-existing or already diagnosed as chronic or permanent $\mathrm{AF}$.

We analyzed the presence of pleural effusion diagnosed by chest $\mathrm{X}$ ray and thoracic ultrasound.

The values of NT pro-BNP was measured during the admission to the emergency department or within the first two days of hospitalization.

We also analyzed, when detectable, the causes of destabilization that had precipitated the HF.

\section{Results}

The total hospitalized admissions with diagnosis of HF were 299. The total number of patients was 238 (108 male and 130 female; mean age 81 years old). Patients hospitalized two or more times were 38 ( $16 \%$ of the total population). Patients with preserved LVEF were 145 (61\% of the total population) including 59 male and 86 female. Patients with reduced LVEF were
93 (39\% of the total population) including 49 male and 44 female (Figure 1).

Among the underlying heart disease: patients with ischemic heart disease were 51 ( $21 \%$ of the total), those with hypertensive heart disease 166 (69 of the total), those with ischemic-hypertensive heart disease 40 (17\% of the total), those with idiopathic dilated cardiomyopathy 12 (5\% of the total) (Figure 2). Patients with valvular disease of at least moderate severity were 90 (38\% of the total) of which 24 with aortic stenosis, 57 with mitral regurgitation, 4 with mitral stenosis and 5 with aortic regurgitation.

Among comorbidities we found 211 patients with hypertension ( $88 \%$ of the total), 68 with diabetes mellitus ( $28 \%$ of the total), 66 with COPD ( $28 \%$ of the total), 112 with anemia ( $47 \%$ of the total) and 99 with renal failure (41\% of the total) (Figure 3).

Patients with permanent AF were 102 approximately $43 \%$ of the total (Figure 3 ).

Patients with pleural effusion were 108 (46\% of

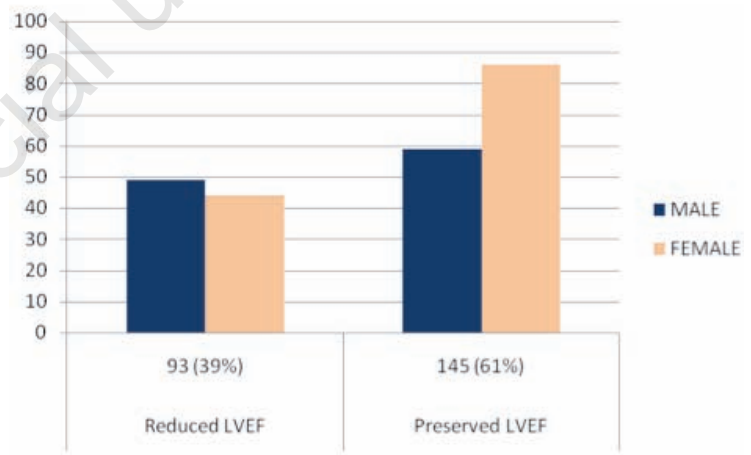

Figure 1. Distribution of the population according to sex and systolic function. LVEF, left ventricular ejection fraction.

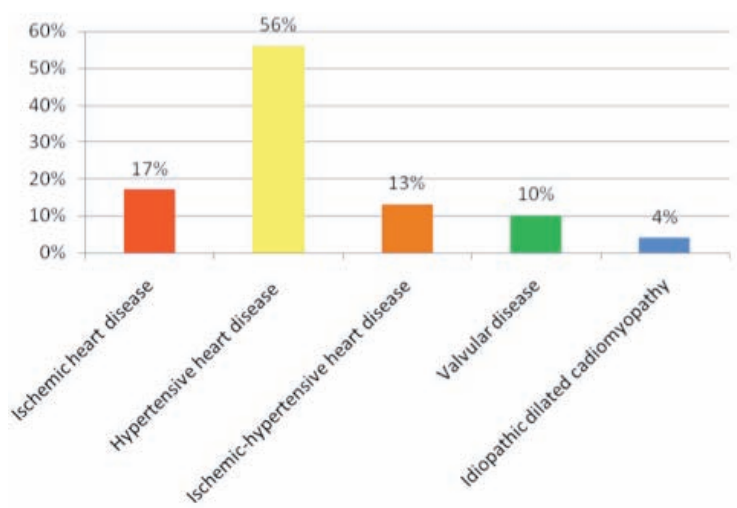

Figure 2. Percentage distribution of the population according to underlying heart disease. 
the total) of which 74 had a bilateral pleural effusion (Figure 4).

The mean value of NT pro-BNP result $6425 \mathrm{pg} / \mathrm{mL}$ in patients with preserved LVEF and $8670 \mathrm{pg} / \mathrm{mL}$ in patients with reduced LVEF.

The cause of destabilization was indeterminate in $50 \%$ of patients. The detected cause was distributed as follows: $13 \%$ infections, $10 \%$ arrhythmias, $10 \%$ acute exacerbation of COPD, $8 \%$ poor compliance, $4 \%$ acute coronary syndrome, $3 \%$ anemia and $2 \%$ acute renal failure.

In the total study population 102 patients (43\% of the total) had 3 to 5 comorbidities, 169 patients (71\% of the total) had at least 2 comorbidities and only 4 patients (1.7\% of the total) had no comorbidity (Figure 5 ).

Rehospitalized patients were 38 (16\% of the total), with an average age of 83 years. $50 \%$ of these patients had a reduced LVEF (19 patients), $68 \%$ had 3 to 5 comorbidities and $89 \%$ had at least two comorbidities. Any patient had no comorbidity. The average values of NT pro-BNP were $10,619 \mathrm{pg} / \mathrm{mL}$ in patients with preserved LVEF and 13,975 in patients with reduced LVEF.

\section{Discussion}

Epidemiological data regarding HF are often ambiguous mainly due to the heterogeneity of the populations studied (hospital statistics, longitudinal or transverse community analysis, clinical trials) and also the lack of a widely accepted definition of HF. In addition, many studies do not allow estimating the actual complexity of the patient suffering from HF (comorbidities and older age are often exclusion criteria from the analysis). In our study we wanted to check whether the literature data could find a match in a real population of patients admitted with diagnosis of HF in a Department of Internal Medicine. We therefore performed a retrospective analysis using the tool of the computerized medical records that allowed us to easily find a vast amount of reliable data regarding the patients examined. In many cases there were also the checklist for diseases whose compilation was scheduled for the most frequently encountered diseases (HF, COPD, stroke, pneumonia, gastrointestinal bleeding). The first observation that emerges from the analysis is that our population is considerably older than the populations considered in most works in the literature including the study TEMISTOCLE ${ }^{19}$ with $89 \%$ of patients older than 70 years of (in TEMISTOCLE study the percentage of patients older than 70 years admitted to medical division for HF was $76.2 \%$ ). Conversely, there was a distribution between gender which provides a slight predominance of the female sex. The highest prevalence of patients with preserved LVEF (61\% in our study compared to $40.8 \%$ in the Medicine-subpopulation of TEMISTO-
CLE study), of hypertensive heart disease (69\% in our study compared to $45.5 \%$ in CONFINE study) and of hypertension ( $88 \%$ in our study compared to $62.8 \%$ in CONFINE study) appears to be justified only by the more advanced average age of our population and by the typical profile of HF with preserved systolic function. With regard to comorbidities, if we compare data obtained from our population with

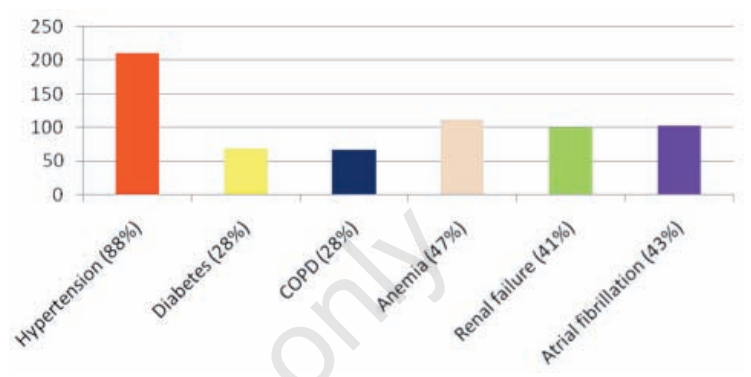

Figure 3. Comorbidity in the general population (absolute number and percentage). COPD, chronic obstructive pulmonary disease.

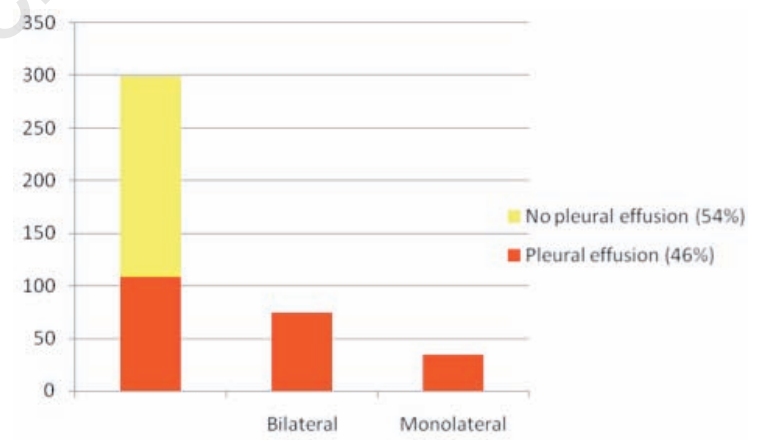

Figure 4. Distribution of the population according to the presence of pleural effusion (monolateral or bilateral).

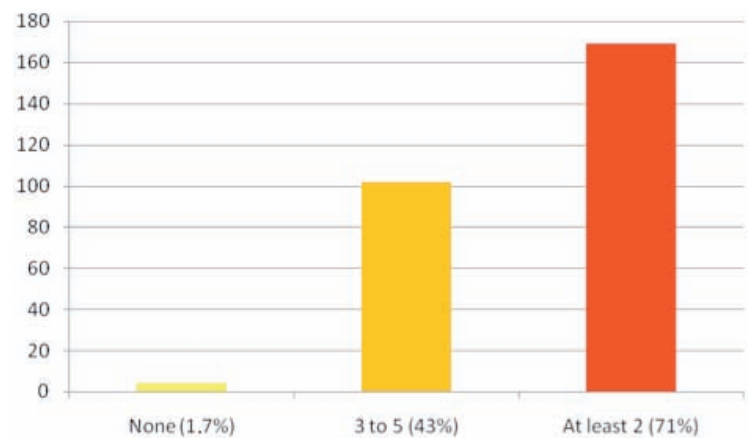

Figure 5. Number of comorbidities (absolute number and percentage). 
those of other studies such as the study CONFINE, ${ }^{20}$ we notice a lower percentage of patients without comorbidity (only $1.7 \%$ in our study compared to the $10 \%$ in the CONFINE study) and significantly higher percentage of patients with two or more comorbidities ( $43 \%$ in our study). The permanent atrial fibrillation in our population showed a prevalence comparable to that reported in the literature. One interesting observation that emerges from our study is the high prevalence $(45 \%)$ of pleural effusion ( $68 \%$ bilateral pleural effusion). This finding may be useful in the differential diagnosis of HF also because of the ease of detection through the use of ultrasound. The pro-BNP was used primarily to exclude the diagnosis of $\mathrm{HF}$ although we detected average values of pro-BNP significantly higher in rehospitalized patients with reduced systolic function. Retrospective analysis did not provide significant data about the causes of destabilization but showed a high prevalence of indeterminate cause. By analyzing rehospitalized patients separately we noted a higher prevalence of comorbidities ( $89 \%$ of patients with at least two comorbidities) and average values of pro-BNP significantly higher than the rest of the population.

The collected data confirm the complexity of patients with HF, often due to advanced age and a high number of comorbidities. These findings are not very different from what has already been demonstrated in previous epidemiological studies on HF as those previously mentioned. ${ }^{19,20}$ In order to compare the collected data with those of other multicenter studies it is right to point out some differences in the method of data collection. In our single-center study we were able to define the comorbidities with greater accuracy (e.g., diagnosis of COPD made only with spirometric tests according to the criteria of the GOLD project, diagnosis of renal failure according to NKF/DOQI). The percentage of COPD, even excluding patients without a diagnosis made by spirometry, was anyway higher than that found in other studies $(28 \%$ in our study compared to $26.7 \%$ in the CONFINE study). In our study, in addition to considering comorbidity taken by itself, we also evaluated the number of comorbidities present simultaneously in hospitalized patients with HF. This index allowed us to highlight more strongly the complexity of patients with HF. Separate analysis of rehospitalized patients (which noted a greater number of comorbidities simultaneously present) confirmed that the greater presence of a lot of comorbidities makes more complex the management of patients with HF. In this sense, it would be interesting to evaluate in patients with $\mathrm{HF}$ a score of comorbidities in order to select patients with higher risk as that require more complex management (e.g., follow-up, more attention in the treatment of single comorbidity).

\section{Conclusions}

Much progress has been made in recent years in the diagnosis and treatment of patient with HF and this is confirmed by literature data showing increased survival of patients with HF. ${ }^{21}$ However, the comparison with the real world shows us a population of patients with different characteristics compared with those provided by the majority of epidemiological studies and clinical trials in which the advanced age and the presence of comorbidities represent criteria for exclusion from the study. The longer life-expectancy of all the major industrialized countries such as ours, as is clear from our study, results in an higher prevalence of a profile of HF patients who have an advanced average age and a higher number of comorbidities. We therefore confirm the usefulness of the systematic data collection on real populations and the creation of registries of pathology in order to have a more truthful picture of HF patients. ${ }^{22}$ This mode has not only an epidemiological purpose but it is useful for both a correct diagnostic and prognostic classification as well as for management of therapies. In fact the presence of several comorbidities makes more difficult the management and the physician must carefully balance advantages and disadvantages of the different drugs within each comorbidity and the HF. If we just think of the implications of renal failure in the proper management of diuretic therapy and of the use of certain medications such as ACE-I and blockers of the RAAS. ${ }^{23}$ Similarly the presence of anemia, often associated with chronic renal failure, represents a condition of refractoriness to treatment for $\mathrm{HF}^{24} \mathrm{COPD}$, which is also a very common comorbidity in our population ( $28 \%$ of total), may create difficulties in the diagnosis of HF and also in the management of therapies (e.g., $\beta$-blockers and $\beta$-stimulants) ${ }^{25}$ In the differential diagnosis of dyspnea we must consider that there is a high prevalence of COPD in patients with HF such as of HF in COPD patients. ${ }^{26}$ For this reason it is important in all patients with dyspnea to have a comprehensive approach that includes, in addition to the examinations of the first level, echocardiogram and spirometry. Therefore it is necessary a comprehensive approach to the patients with HF to highlight and also treat the associated comorbidities, bearing in mind that often the management of various therapies can be complex. The increase in prevalence of subject with HF of older age and with a high number of comorbidities could justify the fact that the rate of rehospitalization of patients with HF does not appear to have significantly decreased in recent years despite the progress made in terms of diagnosis and treatment. ${ }^{27} \mathrm{~A}$ greater understanding of the characteristics of real population of patients suffering from HF will therefore be useful for a better classification of the patient and to calibrate the therapy with the purpose to 
optimize the treatment and reduce rehospitalization. Our study demonstrates how HF can be more and more a reason for hospitalization and consequently of health care. In a population of patients with an increasing average age the role of comorbidity is more important. All this requires us to provide a careful personalization of treatment and avoid a straightforward application of the guidelines.

\section{References}

1. Dickstein K, Cohen-Solal A, Filippatos G, et al. ESC guidelines for the diagnosis and treatment of acute and chronic heart failure 2008: the Task Force for the diagnosis and treatment of acute and chronic heart failure 2008 of the European Society of Cardiology. Developed in collaboration with the Heart Failure Association of the ESC (HFA) and endorsed by the European Society of Intensive Care Medicine (ESICM). Eur J Heart Fail 2008; 10:933-89.

2. Mosterd A, Hoes AW. Clinical epidemiology of heart failure. Heart 2007;93:1137-46.

3. Italian National Health System; Analisi dei ricoveri per insufficienza cardiaca in Italia. Anni 2001-2003; 2007, April 1. Available from: http://www.salute.gov.it

4. Henkel DM, Redfield MM, Weston SA, et al. Death in heart failure: a community perspective. Circ Heart Fail 2008;1:91-7.

5. Maron BJ, Towbin JA, Thiene G, et al. Contemporary definitions and classification of the cardiomyopathies: an American Heart Association Scientific Statement from the Council on Clinical Cardiology, Heart Failure and Transplantation Committee; Quality of Care and Outcomes Research and Functional Genomics and Translational Biology Interdisciplinary Working Groups; and Council on Epidemiology and Prevention. Circulation 2006;113:1807-16.

6. Hogg K, Swedberg K, McMurray J. Heart failure with preserved left ventricular systolic function; epidemiology, clinical characteristics, and prognosis. J Am Coll Cardiol 2004;43:317-27.

7. Lam CS, Donal E, Kraigher-Krainer E, Vasan RS. Epidemiology and clinical course of heart failure with preserved ejection fraction. Eur J Heart Fail 2011;13: 18-28.

8. Mant J, Doust J, Roalfe A, et al. Systematic review and individual patient data meta-analysis of diagnosis of heart failure, with modelling of implications of different diagnostic strategies in primary care. Health Technol Assess 2009; 13:1-207.

9. Kelder JC, Cramer MJ, van Wijngaarden J, et al. The diagnostic value of physical examination and additional testing in primary care patients with suspected heart failure. Circulation 2011;124:2865-73.

10. Rutten FH, Moons KG, Cramer MJ, et al. Recognising heart failure in elderly patients with stable chronic obstructive pulmonary disease in primary care: cross sectional diagnostic study. BMJ 2005;331:1379.

11. Marwick TH, Raman SV, Carrio I, Bax JJ. Recent developments in heart failure imaging. JACC Cardiovasc Imaging 2010;3:429-39.
12. Maisel A, Mueller C, Adams K Jr, et al. State of the art: using natriuretic peptide levels in clinical practice. Eur J Heart Fail 2008;10:824-39.

13. Pocock SJ, Wang D, Pfeffer MA, et al. Predictors of mortality and morbidity in patients with chronic heart failure. Eur Heart J 2006;27:65-75.

14. Ketchum ES, Levy WC. Establishing prognosis in heart failure: a multimarker approach. Prog Cardiovasc Dis 2011;54:86-96.

15. Stewart S, Jenkins A, Buchan S, et al. The current cost of heart failure to the National Health Service in the UK. Eur J Heart Fail 2002;4:361-71.

16. Fihn SD, Gardin JM, Abrams J, et al. 2012 ACCF/AHA/ ACP/AATS/PCNA/SCAI/STS Guideline for the diagnosis and management of patients with stable ischemic heart disease: a report of the American College of Cardiology Foundation/American Heart Association Task Force on Practice Guidelines, and the American College of Physicians, American Association for Thoracic Surgery, Preventive Cardiovascular Nurses Association, Society for Cardiovascular Angiography and Interventions, and Society of Thoracic Surgeons. J Am Coll Cardiol 2012;60: e44-164.

17. Frohlich ED, Apstein C, Chobanian AV, et al. The heart in hypertension. N Engl J Med 1992;327:998-1008.

18. Richardson P, McKenna W, Bristow M, et al. Report of the 1995 World Health Organization/International Society and Federation of Cardiology Task Force on the Definition and Classification of cardiomyopathies. Circulation 1996;93:841-2.

19. Di Lenarda A, Scherillo M, Maggioni AP, et al. Current presentation and management of heart failure in cardiology and internal medicine hospital units: a tale of two worlds-the TEMISTOCLE study.; TEMISTOCLE Investigators. Am Heart J 2003;146:e12.

20. Biagi P, Gussoni G, Iori I, et al. Clinical profile and predictors of in-hospital outcome in patients with heart failure: the FADOI “CONFINE” Study. Int J Cardiol 2011; 152:88-94.

21. Levy D, Kenchaiah S, Larson MG, et al. Long-term trends in the incidence of and survival with heart failure. N Engl J Med 2002;347:1397-402.

22. Fabbri G, Gorini M, Maggioni AP, et al. Italian Network on Congestive Heart Failure: ten-year experience. G Ital Cardiol 2006; 7:689-94.

23. Ronco C, McCullough P, Anker SD, et al. Cardio-renal syndromes: report from the consensus conference of the acute dialysis quality initiative. Eur Heart J 2010;31:703-11.

24. Van Veldhuisen DJ, Anker SD, Ponikowski P, Macdougall IC. Anemia and iron deficiency in heart failure: mechanisms and therapeutic approaches. Nat Rev Cardiol 2011;8:485-93.

25. Dungen HD, Apostolovic S, Inkrot S. Titration to target dose of bisoprolol vs. carvedilol in elderly patients with heart failure: the CIBIS-ELD trial. Eur J Heart Fail 2011;13:670-80.

26. Nozzoli C, Mathieu G, Gensini G. BPCO e scompenso cardiaco. Ital J Med 2011;5:22-37.

27. Ross JS, Chen J, Lin Z, et al. Recent national trends in readmission rates after heart failure hospitalization. Circ Heart Fail 2010;3:97-103. 